\title{
A Cell-based Regional Evacuation Model with Contra-flow Lane
}

\section{Deployment}

\author{
Zhang Xinming ${ }^{1, a}$, An Shi ${ }^{1, b}$ and Xie Binglei ${ }^{2, c}$ \\ ${ }^{1}$ School of Transportation Science and Engineering, Harbin Institute of Technology, Harbin, China \\ ${ }^{2}$ School of Urban Planning and Management, Harbin Institute of Technology Shenzhen Graduate \\ School, Shenzhen, China \\ azxinming@yahoo.cn, banshi@hit.edu.cn, ’xiebinglei@126.com
}

Keywords: regional traffic evacuation, DTA, cell, one-destination evacuation, contra-flow lane

\begin{abstract}
In this paper, a traffic evacuation model is proposed with a framework of SO-DTA model and one-destination evacuation embedded. The network is described as cells and nodes, so that a regional traffic evacuation model which embeds CTM and point-queue is proposed. This paper also optimized the deployment strategy of the contra-flow lane in the premise of system optimization. In the end, a numerical experiment is conducted to analysis the effectiveness of the model. Compared with the CTM, the model constructed reduced the gross evacuation time and the clear time of the road net, and solved the unnecessary "traffic holding" problem.
\end{abstract}

\section{Introduction}

With the developing and expanding of cities, security issues are potential threatened by various disasters. When the degree of disasters reach some threshold level, an emergency evacuation is necessary in order to avoid or reduce the loss of life and property. Transportation plays an important role in evacuation. Constructing models is the core of an evacuation.

In previous studies, the Cell Transmission Model (CTM), proposed by Ziliaskopoulos ${ }^{[1]}$, is considered to be a better basic model for evacuation. But the "traffic holding" problem often happens in this model and its cell-connector topology structure increases the constraints. In part, these observations motivated our study

The first and primary goal of this paper is to construct a cell-based regional evacuation model, which embeds the idea of one-destination evacuation. And using point-queue model, the road network is converted to cell-node topology structure to reduce constraints. The "traffic holding" problem also be solved in the model by an OSP constraint.

Secondly, based on the model proposed, the deployment strategy of contra-flow lane in the premise of system optimization is also optimized in this paper to improve the efficiency of evacuation.

Finally, a numerical experiment is conducted to verify the proposed model.

\section{Literature review}

Existing regional evacuation models can be classified into two basic models: network flow models and dynamic traffic assignment models.

The traditional graph theory is the basic theory of network flow models. The nodes and arcs are used to represent generating points and roads in network flow models ${ }^{[2]}$. Yamada considered the regional evacuation as a minimal cost flow problem, aimed to minimize the driving distance ${ }^{[3]}$. 
Cova and Johnson believed that delays in regional evacuation often occurred at intersections and presented a network flow model for identifying optimal lane-based evacuation routing plans in a complex road network. The model is an integer extension of the minimum cost flow problem ${ }^{[4]}$.

SO-DTA has been recognized to be a better basic model to solve evacuation problems. Ziliaskopoulos brought CTM to SO-DTA, but limited to only one destination ${ }^{[1]}$. Han proposed the concept of one-destination evacuation which could significantly simplified evacuation problem $^{[5]}$.

Each model has its advantages and disadvantages. Network flow models describe the traffic flow using graph vividly, but it cannot effectively reflect some dynamic characteristics of traffic flow, such as queues. Most agree that SO-DTA based on CTM could be a better model for evacuation. But the model is limited to only one destination in evacuation. When more destinations exist, it would become complex. One-destination evacuation ${ }^{[6]}$, proposed by Han, can solve the problem suitably.

There is another problem in CTM. Under the premise of system optimization, the "traffic holding" problem often happens at interactions. Some "traffic holding" is necessary but some is unnecessary. Unnecessary "traffic holding" is hardly understood by evacuees. The model this paper proposed can solve the problem.

\section{A cell-based regional evacuation model}

\subsection{One-destination evacuation network}

In daily traffic, every person has his own destination. While in evacuation, the primary purpose is to reach the shelters as soon as possible. It is not important that which paths or shelters should be chosen. So Han assumed that there was a most-desirable-destination, that is, one-destination evacuation $^{[5]}$.

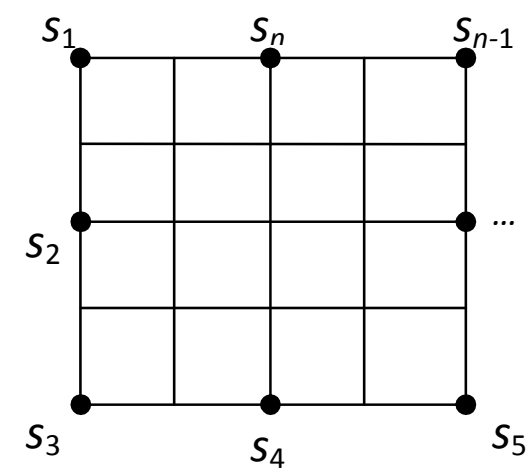

(a) The original multiple-destination network

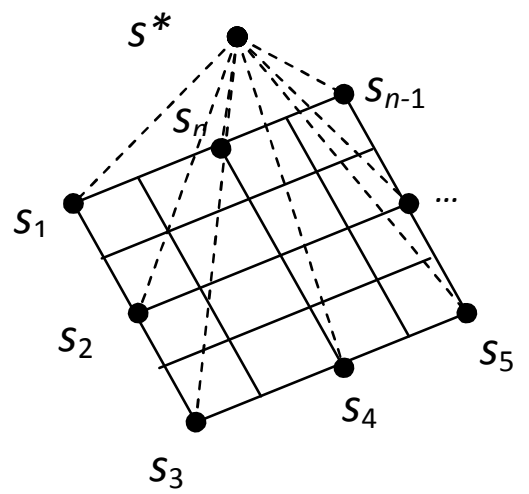

(b) The one-destination network

Figure 1: The network transformation

In Figure 1(a), there are $m$ origins and $n$ destinations in the evacuation. In previous studies, we will have an O-D matrix with $m$ rows and $n$ columns. Strictly speaking, when we get the O-D matrix by the Shortest Path Method or other methods, the system optimization is not considered. Han added a "dummy-destination point", denoted as $S^{*}$, to the network, as shown in Figure 1(b). Then "dummy links" were used to lead from each real-world destinations to $S^{*}$. It is assumed that all these dummy links have infinite capacity and zero cost. Thus the O-D matrix has been simplified to $m$ rows and only one column. At the same time, destinations and paths are chosen synchronously. This is consistent with the system optimization.

\subsection{A cell-based regional evacuation model}

Consider a network $G(N, A)$, where $N$ and $A$ are the sets of nodes and links respectively. Let $R$ represent the set of origins and $s^{*}$ the single destination. The assignment horizon is divided into a finite number of discrete time intervals indexed by $\{t: t=0,1, \ldots, T\}$. 
Let $O(i)$ and $I(i)$ be the sets of outgoing and incoming cells of node $i \in N$, Let $Q_{r s} *(t)$ be the travel demand between O-D pair $r$-s* departing at time $t$, and the numbers of vehicles on, entering, and exiting a link $a$ at time $t$ are denoted by $X_{a}(t), U_{a}(t)$ and $V_{a}(t)$ respectively.

Merchant and Nemhauser constructed a basic dynamic traffic assignment model in 1978, Carey modified the model as follows ${ }^{[7]}$ :

$$
\begin{array}{ll} 
& \min \sum_{t=1}^{T} \sum_{a \in A} X_{a}(t) \\
\text { s.t. } & X_{a}(t+1)=X_{a}(t)-V_{a}(t)+U_{a}(t) \\
& \sum_{a \in O(i)} U_{a}(t)=Q_{i s^{*}}(t)+\sum_{a \in I(i)} V_{a}(t) \\
& X_{a}(0)=R_{a} \geq 0 \\
& X_{a}(t), V_{a}(t), U_{a}(t) \geq 0
\end{array}
$$

Constraint (1b) describes traffic flow propagation; Constraint (1c) represents node flow conservation; Constraint (1d) is the initial condition while (1e) is nonnegative constraint.

Note that the model above does not reflect the queue. Thus we embed the point-queue model into M-N model. So at time $t$, we can divide the numbers of vehicles on a link $a$ into two parts: the queued flow $P_{a}(t)$ at the end of time $t$ and the exit flow $V_{a}(t)$ during time $t$. That is,

$$
X_{a}(t)=P_{a}(t)+V_{a}(t)
$$

Then the model can be written as:

$$
\begin{array}{ll} 
& \min \sum_{t=1}^{T} \sum_{a \in A}\left(P_{a}(t)+V_{a}(t)\right) \\
\text { s.t. } & P_{a}(t+1)=P_{a}(t)-V_{a}(t+1)+U_{a}(t) \\
& \sum_{a \in O(i)} U_{a}(t)=Q_{i s^{*}}(t)+\sum_{a \in I(i)} V_{a}(t) \\
& P_{a}(0)+V_{a}(0)=R_{a} \\
& U_{a}(t) \geq 0 \\
& 0 \leq V_{a}(t) \leq V_{a m} \\
& P_{a}(t)>0 \Rightarrow V_{a}(t)=V_{a m}
\end{array}
$$

Where $V_{a m}$ is the maximum outflow of a link $a$. Constraint (3g) requires the link to be operated at capacity at $t$ when a queue is present at the end of $t$. That is the OSP constraint. Violating this condition thus implies "holding" flows when there is still spare capacity.

In the model 3, the queue has been reflected but it cannot propagate. Learning from the CTM, we transform the original network into a cell-node network. Thus link $a$ is divided into several cells so that we can use cell $a$ instead. According CTM,

$$
\begin{aligned}
& U_{a}(t) \leq U_{a m} \\
& U_{a}(t) \leq \omega / v_{f}\left[N_{j}(k)-X_{j}(k)\right]=\omega / v_{f}\left[H_{a}-P_{a}(t)-V_{a}(t)\right]
\end{aligned}
$$

Where $U_{a m}$ is the maximum inflow of a cell $a$ and $H_{a}$ is the maximum vehicles cell $a$ can hold. Let $a^{+}$be the downstream node of cell $a$. Then the OSP constraint can be written as

$$
\begin{aligned}
P_{a}(t)>0 & \Rightarrow V_{a}(t)=V_{a m}, \\
& \operatorname{or} U_{a^{+}}(t)=U_{a^{+} m}, \\
& \operatorname{or} U_{a^{+}}(t)=\omega / v_{f}\left[H_{a^{+}}-P_{a^{+}}(t)-V_{a^{+}}(t)\right]
\end{aligned}
$$

Adding the constraint of the holding capacities of real destinations, we can obtain a cell-based regional evacuation model as follows:

$$
\min \sum_{t=1}^{T} \sum_{a \in A}\left(P_{a}(t)+V_{a}(t)\right)
$$


s.t. [Original Constraints](3b-3f)

$U_{a}(t) \leq \omega / v_{f}\left[H_{a}-P_{a}(t)-V_{a}(t)\right]$

$\sum_{i=1}^{T} U_{l_{i}}(t) \leq H_{S_{i}}$

[OSP Constraints](5)

3.3 The contra-flow lane deployment

Let cell $a$ and cell $-a$ are the two cell of a link with opposite directions. The two have the same characteristic except direction. Let $v_{a m}\left(u_{a m}\right)$ is the capacity of a lane in cell $a, h_{a}$ is the holding capacity of a lane in cell $a, n_{a}$ is the number of lane that cell $a$ have and $\rho_{a}$ is the number of contra-flow lanes in cell $a$. If $\rho_{a}<0$, it means $\left|\rho_{a}\right|$ contra-flow lanes are needed in cell $-a$.

When contra-flow lanes are deployed on cell $a$, the capacity $V_{a m}$ and the holding capacity $H_{a}$ of cell $a$ are changed as Table 1 .

Table 1 The capacity $V_{a m}$ and the holding capacity $H_{a}$ of cell a changed

\begin{tabular}{ccccc}
\hline cell & $V_{a m}$ before & $V_{a m}$ after & $H_{a}$ before & $H_{a}$ after \\
\hline$a$ & $V_{a m}$ & $V_{a m}-\rho_{a} * v_{a m}$ & $H_{a}$ & $H_{a}-\rho_{a} * h_{a}$ \\
$-a$ & $V_{-a m}$ & $V_{-a m}+\rho_{a} * v_{a m}$, & & $H_{-a}+\rho_{a} * h_{a}$, \\
& or $V_{-a m}-\rho_{-a} * v_{-a m}$ & $H_{-a}$ & or $H_{-a}-\rho_{-a} * h_{-a}$ \\
\hline
\end{tabular}

Then the model can be shown as follows:

$$
\min \sum_{t=1}^{T} \sum_{a \in A}\left(P_{a}(t)+V_{a}(t)\right)
$$

s.t. [Original Constraints](3b-3f)

[Holding Capacity Constraints](6d)

$V_{a}(t) \leq V_{a m}-\rho_{a} * v_{a m}$

$$
\begin{aligned}
& U_{a}(t) \leq U_{a m}-\rho_{a} * v_{a m} \\
& U_{a}(t) \leq \omega / v_{f}\left[H_{a}-\rho_{a} * h_{a}-P_{a}(t)-V_{a}(t)\right]
\end{aligned}
$$

$P_{a}(t)>0 \Rightarrow V_{a}(t)=V_{a m}-\rho_{a} * v_{a m}$,

$$
\operatorname{or} U_{a^{+}}(t)=U_{a^{+} m}-\rho_{a^{+}} * v_{a^{+} m}
$$$$
\operatorname{or} U_{a^{+}}(t)=\omega / v_{f}\left[H_{a^{+}}-\rho_{a^{+}} * h_{a^{+}}-P_{a^{+}}(t)-V_{a^{+}}(t)\right]
$$

$-n_{a} \leq \rho_{a} \leq n_{a}, \quad \rho_{a}$ is an integer

$\rho_{-a}+\rho_{a}=0$

\subsection{Algorithm}

Noted that without thinking constraint $7 \mathrm{~g}$ (OSP constraint), model 7 is an integer programming model, we can use traditional algorithm to solve the model. Thinking about OSP constraint, Ho designed an efficient algorithm in $1980^{[8]}$. We improve it and design a new algorithm as follows:

Step 0: Solve the integer programming problem to get the optimal system $\operatorname{cost} Z_{T}{ }^{*}$. Set $w=T$.

Step 1: Test the OSP constraint, stop if satisfied; otherwise, go to Step 2.

Step 2: Solve the following integer programming problem and get $E_{w-1}{ }^{*}$.

$$
\max E_{w-1}=\sum_{t=1}^{w-1} \sum_{a \in A} V_{a}(t)
$$

s.t. $\quad[$ Constraints] $(7 \mathrm{a}-7 \mathrm{f}, 7 \mathrm{~h}, 7 \mathrm{i})$

$$
\sum_{t=1}^{T} \sum_{a \in A}\left(P_{a}(t)+V_{a}(t)\right)=Z_{T}^{*}
$$


Step 3: Set $w=w$-1; test the OSP constraint, stop if satisfied; otherwise, add the following constraint into model 8: $\sum_{t=1}^{w-1} \sum_{a \in A} V_{a}(t)=E_{w-1}^{*}$, and then go back to Step 2.

\section{Numerical example}

A numerical example is briefly described in the following. In Figure 2, There are 12 nodes and 17 links in the network. Each link has 6 lanes. The evacuation demand 1150, 850 and 1000 are loaded into the network by three points-O1, O2, and $\mathrm{O} 3$ respectively. $\mathrm{J}$ and $\mathrm{K}$ are the real destinations with 2000 holding capacities both. Point $\mathrm{s}$ is the dummy-destination.
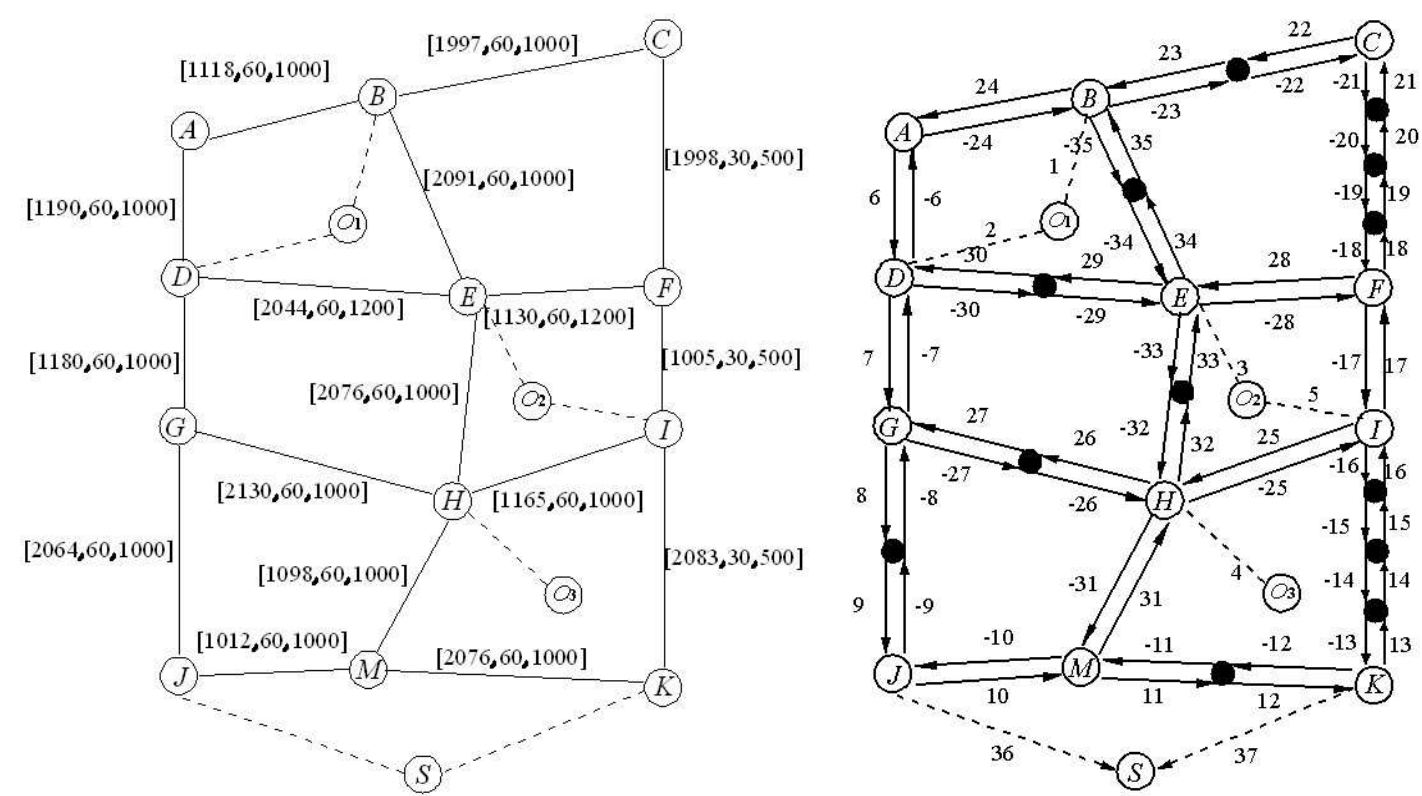

(a) Original network[length $(\mathrm{m})$, speed $(\mathrm{km} / \mathrm{h})$, capacity $(\mathrm{veh} / \mathrm{h})](\mathrm{b})$ cell-node network

Figure 2 The evacuation network:

The analysis period is 30 minutes, divided into 30 time intervals. The model is solved using "lingo" and the result we get is that :

(1)The system cost is 22350 minutes and the time for evacuation is 16 minutes.

(2)To improve the efficiency of evacuation, we also can obtain from the result that these links, including GD, JG, KM, JM, KI, IF, FC and MH, need to deploy contra-flow lanes keeping the system optimal.

(3)By analysis the result seriously, "traffic holding" problem do not happen in our model.

(4)Also, we can get that how much demand is loaded into the network every time interval. Like Table 2.

Table 2 Demand loading

\begin{tabular}{ccccccccc}
\hline & $0-1$ & $1-2$ & $2-3$ & $3-4$ & $4-5$ & $5-6$ & $6-7$ & $7-8$ \\
\hline $\mathrm{O}_{1}$ & 360 & 360 & 210 & 150 & 70 & 0 & 0 & 0 \\
$\mathrm{O}_{2}$ & 240 & 210 & 50 & 100 & 50 & 50 & 100 & 50 \\
$\mathrm{O}_{3}$ & 250 & 150 & 200 & 150 & 130 & 100 & 20 & 0 \\
\hline
\end{tabular}

\section{Conclusions}

A new model for evacuation based on cell is proposed in this paper. In the model, we make use of the CTM and point-queue model. Based on the model, the road network is converted to the cell-node topology structure so that the constraints of evacuation model are significantly reduced. The idea of one-destination evacuation makes the feasibility of our model. 
By adding a new constraint, called OSP constraint, the "traffic holding" problem is solved. And we improve the model to get contra-flow lanes deployment in evacuation. This improves the efficiency of evacuation significantly.

In the model proposed, when the network becomes more complex, the solution of the model will be more different. So a more efficient algorithm will be designed in the future study. Besides, it would better if other management of evacuation can be embedded into the model, such as staged evacuation.

\section{Acknowledgements}

This work was partially supported by the NSFC under Grant No. 70973032 and No.71173061.

\section{References}

[1] Ziliaskopoulos A K. A Linear Programming Model for The Single Destination System Optimum Dynamic Traffic Assignment Problem[J]. Transportation Science, 2000,34(1):37-49.

[2] Hamacher H W, Tjandra S A. Mathematical Modeling of Evacuation Problems - a state of art[C]. Pedestrian and Evacuation Dynamics, 2001:59-74.

[3] Yamada T. A Network Flow Approach to A City Emergency Evacuation Planning[J]. International Journal of Systems Science, 1996, 27(10):931-936.

[4] Cova T J, Johnson J P. A Network Flow Model for Lane-based Evacuation Routing[J]. Transportation Research Part A: Policy and Practice, 2003, 37(7):579-604.

[5] Han D L. Evacuation Modeling and Operations Using Dynamic Traffic Assignment and Most-desirable-destination Approaches[C]. Washington D.C.: The 84th Transportation Research Board Annual Meeting, 2005:964-969.

[6] Han D L. Evacuation Modeling and Operations Using Dynamic Traffic Assignment and Most-desirable-destination Approaches[C]. Washington D.C.: The 84th Transportation Research Board Annual Meeting, 2005:964-969.

[7] Carey M. Optimal Time-varying Flows on Congestion Networks[J]. Operations Research, 1987, 35(1):58-69.

[8] Ho J K. A Successive Linear Optimization Approach to The Dynamic Ttraffic Assignment Problem[J]. Transportation Science, 1980, 14(4):295-305. 